\title{
The Nonequilibrium Thermodynamics of Small Systems
}

\begin{abstract}
The interactions of tiny objects with their environments are dominated by thermal fluctuations. Guided by theory and assisted by new micromanipulation tools, scientists have begun to study such interactions in detail.
\end{abstract}

\section{Carlos Bustamante, Jan Liphardt, and Felix Ritort}

mall systems found throughout physics, chemistry, and biology manifest striking properties as a result of their tiny dimensions. Examples of such systems include magnetic domains in ferromagnets, which are typically smaller than $300 \mathrm{~nm}$; quantum dots and biological molecular machines that range in size from 2 to $100 \mathrm{~nm}$; and solidlike clusters that are important in the relaxation of glassy systems and whose dimensions are a few nanometers. Scientists nowadays are interested in understanding the properties of such small systems. For example, they are beginning to investigate the dynamics of the biological motors responsible for converting chemical energy into useful work in the cell (see the article by Terence Strick, JeanFrançois Allemand, Vincent Croquette, and David Bensimon, PHYSICS TODAY October 2001, page 46). Those motors operate away from equilibrium, dissipate energy continuously, and make transitions between steady states.

Until the early 1990s, researchers had lacked experimental methods to investigate such properties of small systems as how they exchange heat and work with their environments. The development of modern techniques of microscopic manipulation has changed the experimental situation. In parallel, during the past decade, theorists have developed several results collectively known as fluctuation theorems (FTs), some of which have been experimentally tested. The much-improved experimental access to the energy fluctuations of small systems and the formulation of the principles that govern both energy exchanges and their statistical excursions are starting to shed light on the unique properties of microscopic systems. Ultimately, the knowledge physicists are gaining with their new experimental and theoretical tools may serve as the basis for a theory of the nonequilibrium thermodynamics of small systems.

\section{Molecular machines}

Thermodynamics describes energy exchange processes of macroscopic systems: Objects as varied as liquids, magnets, superconductors, and even black holes comply with its laws. In macroscopic systems, behavior is reproducible and fluctuations (deviations from the typically observed, average behavior) are small. It is only under some special

Carlos Bustamante is a Howard Hughes Medical Institute investigator and a professor of molecular and cell biology, chemistry, and physics at the University of California, Berkeley. Jan Liphardt is an assistant professor of biophysics, also at the University of California, Berkeley. Felix Ritort is a professor of statistical physics at the University of Barcelona in Spain. conditions that thermal fluctuations produce readily detectable consequences in macroscopic systems. Wellknown examples include the opalescence of light in a fluid at its critical point and the blue color of the sky, which is a result of light scattering.

As a system's dimensions decrease, fluctuations away from equilibrium begin to dominate its behavior. In particular, in a nonequilibrium small system, thermal fluctuations can lead to observable and significant deviations from the system's average behavior. Therefore, such systems are not well described by classical thermodynamics. Systems of this type abound in the laboratory, where scientists are building motors with dimensions of less than $100 \mathrm{~nm}$ (see figure 1a), and in the cell, where the biological function and efficiency of molecules such as the molecular motor kinesin are determined by molecular size (figure 1b).

Kinesin is one of many molecular machines. In the cell, those machines use the energy of bond hydrolysis to perform useful work such as the replication, transcription, and repair of DNA and the translation of RNA. Kinesin's role is to carry subcellular cargoes along microtubules. On average, a kinesin motor takes one 8-nm step every 10-15 milliseconds. A single adenosinetriphosphate (ATP) molecule is hydrolyzed per step, and the chemical energy released is tightly coupled to movement and force generation. The kinesin is highly processive-that is, it takes many steps before detaching from its microtubule track.

How efficient is the kinesin motor and how much energy does it dissipate as it moves along the track? The chemical energy released by ATP hydrolysis is about $20 k_{\mathrm{B}} T$. (In the world of small systems, the product of Boltzmann's constant and temperature is a convenient energy unit.) The motor does about $12 k_{\mathrm{B}} T$ of work with each step. Thus, the machine's efficiency is roughly $60 \%$ and it dissipates about $650 k_{\mathrm{B}} T$ per second into its environment.

Molecular machines are unlike macroscopic ones in that they can harness thermal fluctuations and rectify them using energy from chemical sources. Consider, for example, RNA polymerase, an enzyme that moves along DNA to produce a newly synthesized RNA strand-a process called transcription. Although it has not yet been proven unequivocally, evidence suggests that during transcription the polymerase moves by extracting energy from the thermal bath and uses bond hydrolysis to ensure that only "forward" fluctuations are captured. That is, the enzyme rectifies thermal fluctuations to directed motion. The amount of energy required to step from one DNA base to another, the shape of the enzyme, the structural roughness of DNA, and the information encoded in the steps (that is, the base sequence of the DNA helix) are all essential aspects that are attributable to the smallness of the system and that ultimately determine its dynamics.

As with macroscopic systems, for small systems one can distinguish between two situations in which the systems' behavior and properties do not change with 

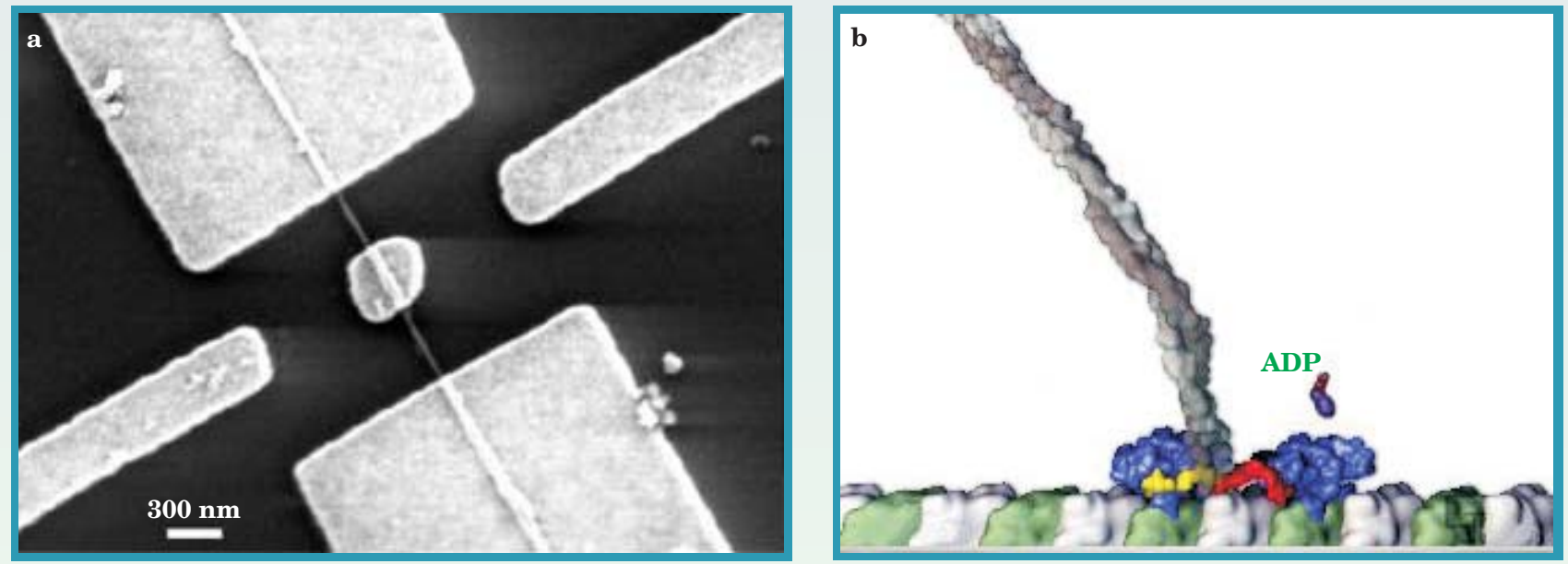

Figure 1. Nonequilibrium small systems. (a) In this scanning electron microscope image of an integrated synthetic actuator, the central metal-plate rotor is attached to a multiwalled carbon nanotube (MWNT) that acts as a support shaft. Electrical contact to the rotor plate is made via the MWNT and its anchor pads. A synchronized electrostatic force can induce rotary motion about the axis of the MWNT. (Adapted from ref. 16.) (b) This artist's rendition, based on crystallographic studies, shows a kinesin motor walking along a microtubule in a hand-over-hand (blue regions) fashion to carry organelles and other cargo from one part of the cell to another. Every step of the motor involves the hydrolytic conversion of chemical energy from adenosinetriphosphate into mechanical work. The reaction cycle is completed with the release of the hydrolysis product, adenosinediphosphate (ADP). Each of the motor's hands is about $5 \mathrm{~nm}$ long.

time: equilibrium states and nonequilibrium steady states. Systems in nonequilibrium steady states have net currents that flow across them, but their properties do not display observable time dependence. Examples of nonequilibrium steady-state systems include an object in contact with two thermal sources at different temperatures, for which the current is a heat flux; a resistor with electric current flowing across it; and the kinesin-microtubule system, for which kinesin motion is the current. Such systems require a constant input of energy to maintain their steady state. Because the systems constantly dissipate net energy, they operate away from equilibrium. Most biological systems, including molecular machines and even whole cells, are nonequilibrium steady states. In a nonsteady-state system, the most general case, one or more of the system's properties change in time.

Figure 2 shows various thermal systems classified according to their size and typical dissipation energy rate, along with a couple of macroscopic systems for comparison. Most of the small systems are characterized by length scales in the nanometer-to-micrometer range and dissipation rates of $10-1000 k_{\mathrm{B}} T / \mathrm{s}$.

\section{The state of a small system}

External variables such as temperature, pressure, and chemical potential specify the different ensembles in statistical mechanics. All such ensembles yield the same equation of state in the large-volume or thermodynamic limit. For small systems, the equation of state and the spectrum of fluctuations are fully determined by so-called control parameters. Figure 3 illustrates two control parameters that may be used to define the state of a small stretched polymer.

By giving direct access to control parameters of single microscopic systems, micromanipulation technology has opened up new opportunities to study nonequilibrium small systems. By varying such parameters, one can perform controlled experiments in which the system is driven away from its initial state of equilibrium and its subsequent response is observed. ${ }^{1}$

Consider, for example, the system illustrated in figure $3 \mathrm{a}$, and imagine that a tethered polymer initially at equilibrium is driven out of equilibrium by the action of an external perturbation that moves the two walls farther apart. In that situation, the control parameter is the distance between the two walls, and the nonequilibrium protocol is fully specified by giving the wall-to-wall distance as a function of time $X(t)$. Since the system is small and is placed in a thermal bath, its dynamics will be effectively random: Even if the nonequilibrium protocol is exactly repeated, the trajectory followed by the system will be different. Each trajectory may be represented by the time evolution of the positions of all atoms $\left\{x_{i}(t)\right\}$. As the control parameter $X$ evolves, the total energy of the system, $U\left(\left\{x_{i}\right\}, X\right)$, varies in a manner that has two distinct contributions,

$$
d U=\sum_{i}\left(\frac{\partial U}{\partial x_{i}}\right)_{X} d x_{i}+\left(\frac{\partial U}{\partial X}\right)_{\left\{x_{i}\right\}} d X=d Q+d W
$$

The first term is the variation of the energy resulting from the change in the internal configuration-that is, a change in heat $Q$-and the second term is the variation of the energy resulting from the perturbation applied while varying the control parameter-that is, a change in work $W$. If the control parameter changes from 0 to $X_{f}$, the total work done on the system is given by

$$
W=\int_{0}^{X_{\mathrm{f}}} d W=\int_{0}^{X_{\mathrm{f}}} F d X
$$

where $(\partial U / \partial X)_{\left\{x_{i}\right\}}$ is the force applied to move the walls. The heat $Q$ exchanged in the nonequilibrium process may be expressed as $\Delta U-W$, where $\Delta U$ is the variation of the energy.

Random fluctuations dominate the thermal behavior in small systems. Since the force is a fluctuating quantity, $W, Q$, and $\Delta U$ will also fluctuate for different trajectories, and the amount of heat or work exchanged with the bath will fluctuate in magnitude and even sign. For a given nonequilibrium protocol, the work and heat probability distributions $P(W)$ and $P(Q)$ characterize the work and heat collected over an infinite number of experiments. In general, those distributions will depend on the details of the experimental protocol. Distributions such as $P(W)$ and $P(Q)$ are important for providing detailed information 
about how a system responds when subjected to a particular experimental process.

\section{Fluctuation theorems}

Nonequilibrium systems are characterized by irreversible heat losses between the system and its environment, typically a thermal bath. Fluctuation theorems embody recent developments toward a unified treatment of arbitrarily large fluctuations in small systems.

In equilibrated, time-reversal-invariant systems, no net heat is transferred from the system to the bath. Therefore the probability of absorbing a given amount of heat must be identical to that of releasing it, and the ratio $P(Q) / P(-Q)$ equals 1 . The probability ratio becomes different from 1 under nonequilibrium conditions. We assume time-reversal invariance, but note that for equilibrated, noninvariant systems-if, for example, magnetic fields are present-it is the total probability for heat absorption that is equated with the total probability for heat release.

The mid-1990s saw the introduction of two important FTs. Denis Evans and Debra Searles derived an FT for systems evolving from equilibrium toward a nonequilibrium steady state, and Giovanni Gallavotti and Eddie Cohen developed an FT for steady-state systems. ${ }^{2}$ The two works were based on numerical evidence obtained previously. ${ }^{3}$ In steady-state systems, an external agent continuously produces heat that is transferred to the bath. The average amount of heat $\langle Q\rangle$ so produced implies an increase in the total average entropy of the system plus environment equal to $\langle S\rangle=\langle Q\rangle / T$.

The rate at which the system exchanges heat with the bath is called the entropy production, $\sigma=Q / T t$, where $t$ is the interval of time over which the system exchanges the heat $Q$. Associated with the entropy production is a timedependent probability distribution $P_{t}(\sigma)$. Gallavotti and Cohen established an explicit mathematical expression that holds under very general conditions for the ratio $P_{t}(\sigma) / P_{t}(-\sigma)$ in steady states,

$$
\lim _{t \rightarrow \infty} \frac{k_{\mathrm{B}}}{t} \ln \left(\frac{P_{t}(\sigma)}{P_{t}(-\sigma)}\right)=\sigma .
$$

Although this expression involves a limit of infinite time, a similar expression without the limit should be valid, to good approximation, as long as $t$ is much greater than the decorrelation time, which is, roughly speaking, the recovery time of the steady state after it is slightly perturbed.

Equation 3 indicates that a steady-state system is more likely to deliver heat to the bath ( $\sigma$ is positive) than it is to absorb an equal quantity of heat from the bath ( $\sigma$ is negative). Nonequilibrium steadystate systems always dissipate heat on average. For macroscopic systems, the heat is an extensive quantity and therefore the ratio of probabilities $P(\sigma) / P(-\sigma)$ grows exponentially with the system's size. That is to say, the probability of heat absorption by macroscopic systems is insignificant.

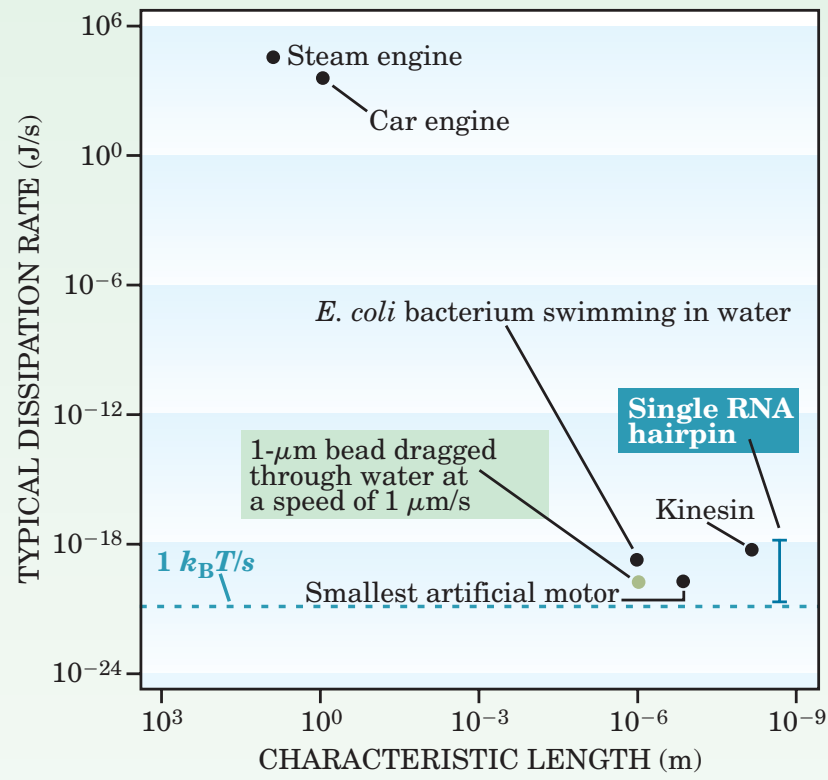

Figure 2. Thermodynamic systems characterized according to their typical length scales and energy dissipation rates. The two systems set off by boxes have been used to test fluctuation theorems and the Jarzynski equality as described in the text.

Our bodies, for example, are maintained in a nonequilibrium state by metabolic processes that dissipate heat all the time. For small systems such as molecular motors that move along a molecular track, however, the probability of absorbing heat can be significant. On average, molecular motors produce heat, but it may be that they move by rectifying thermal fluctuations-a process that would imply the occasional capture of heat from the bath.

Fluctuation theorems shed light on Loschmidt's paradox. In 1876, Josef Loschmidt raised an objection to Boltzmann's derivation of the second law of thermodynamics from Newton's laws of motion. According to Loschmidt, since the microscopic laws of mechanics are invariant under time reversal, there must also exist entropydecreasing evolutions that apparently violate the second law. The FTs show how macroscopic irreversibility arises from time-reversible microscopic equations of motion. Time-reversed trajectories do occur, but they become van- a

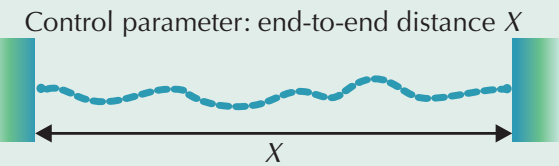

Figure 3. Control parameters for a stretched polymer. (a) The end-toend distance $X$ is the fixed control parameter. In experiments, one can vary $X$ by moving the walls. When $X$ is the control parameter, the force acting on a bead that attaches the polymer to the wall is a fluctuating variable. (b) Here the polymer is fixed at one wall and the control parameter is the force acting on a bead attached to the polymer at its free end. Experimentally, one can fix that force by using a magnetic bead with magnetic moment equal to $\mu$ and applying a uniform external magnetic field gradient $\partial B_{z} / \partial z$. By changing the value of the gradient, one controls the force $F$ acting on the bead. For a fixed $F$, the polymer's extension is a fluctuating variable. In general, the relation between force and extension for a small system whose response is not linear will depend on which variable is the control parameter and which is allowed to fluctuate. (Adapted from ref. 17.)

b

Control parameter: external force

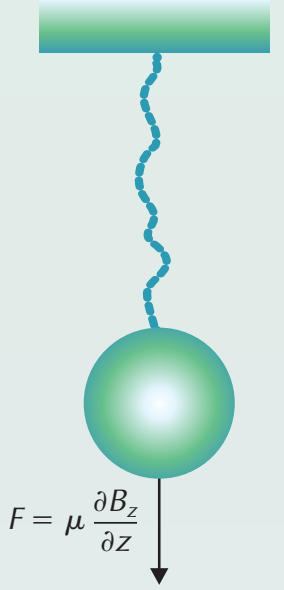




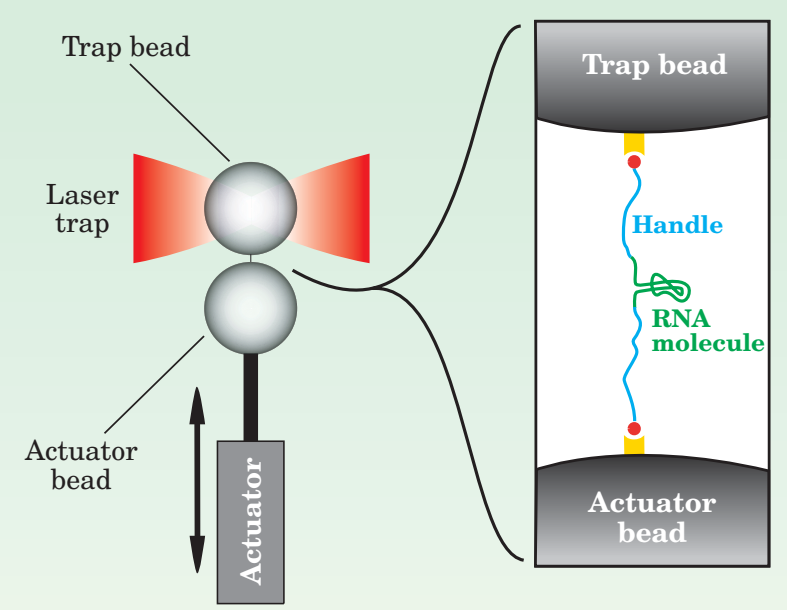

Figure 4. Testing the Jarzynski equality. A molecule of RNA is attached to two beads and subjected to reversible and irreversible cycles of folding and unfolding. A piezoelectric actuator controls the position of the bottom bead, which, when moved, stretches the RNA. An optical trap formed by two opposing lasers captures the top bead, and the change in momentum of light that exits the two-beam trap determines the force exerted on the molecule connecting the two beads. The difference in positions of the bottom and top beads gives the end-to-end length of the molecule. The blowup shows how the RNA molecule (green) is coupled with the two beads via molecular handles (blue). The handles end in chemical groups (red) that can be stuck to complementary groups (yellow) on the bead. The blowup is not to scale: The diameter of the beads is around $3000 \mathrm{~nm}$, much greater than the 20-nm length of the RNA.

ishingly rare with increasing system size. For large systems, the conventional second law emerges.

\section{The Jarzynski equality}

The various FTs that have been reported differ in the details of such considerations as whether the system's dynamics are stochastic or deterministic, whether the kinetic energy or some other variable is kept constant, and whether the system is initially prepared in equilibrium or in a nonequilibrium steady state. A novel treatment of dissipative processes in nonequilibrium systems was introduced in 1997 when Christopher Jarzynski reported a nonequilibrium work relation, ${ }^{4}$ now called the Jarzynski equality (JE). (See PHYSICS TODAY, September 2002, page
19.) The JE indicates a practical way to determine freeenergy differences. Consider a system, kept in contact with a bath at temperature $T$, whose equilibrium state is determined by a control parameter $x$. Initially, the control parameter is $x_{\mathrm{A}}$ and the system is in an equilibrium state $\mathrm{A}$. The nonequilibrium process is obtained by changing $x$ according to a given protocol $x(t)$, from $x_{\mathrm{A}}$ to some final value $x_{\mathrm{B}}$. In general, the final state of the system will not be at equilibrium. It will equilibrate to a state $B$ if it is allowed to further evolve with the control parameter fixed at $x_{\mathrm{B}}$. The JE states that

$$
\exp \left(-\frac{\Delta G}{k_{\mathrm{B}} T}\right)=\left\langle\exp \left(-\frac{W}{k_{\mathrm{B}} T}\right)\right\rangle,
$$

where $\Delta G$ is the free-energy difference between the equilibrium states $\mathrm{A}$ and $\mathrm{B}$, and the angle brackets denote an average taken over an infinite number of nonequilibrium experiments repeated under the protocol $x(t)$. Frequently, the $\mathrm{JE}$ is recast in the form $\left\langle\exp \left(-W_{\mathrm{dis}}\right) / k_{\mathrm{B}} T\right\rangle=1$, where $W_{\text {dis }}=W-\Delta G$ is the dissipated work along a given trajectory.

The exponential average appearing in the JE implies that $\langle W\rangle \geq \Delta G$ or, equivalently, $\left\langle W_{\mathrm{dis}}\right\rangle \geq 0$, which, for macroscopic systems, is the statement of the second law of thermodynamics in terms of free energy and work. An important consequence of the $\mathrm{JE}$ is that, although on average $W_{\text {dis }} \geq 0$, the equality can only hold if there exist nonequilibrium trajectories with $W_{\text {dis }} \leq 0$. Those trajectories, sometimes referred to as transient violations of the second law, represent work fluctuations that ensure the microscopic equations of motion are time-reversal invariant. The remarkable JE implies that one can determine the freeenergy difference between initial and final equilibrium states not just from a reversible or quasi-static process that connects those states, but also via a nonequilibrium, irreversible process that connects them. The ability to bypass reversible paths is of great practical importance.

In 1999, Gavin Crooks related various FTs by deriving a generalized theorem for stochastic microscopically reversible dynamics. ${ }^{5}$ The box below gives details. The past six years have seen further consolidation, and physicists now understand that neither the details of just which quantities are maintained constant during the dynamics nor the somewhat differing interpretations of entropy production, entropy production rate, dissipated work, exchanged heat, and so forth lead to fundamentally distinct FTs.

\section{The Crooks Fluctuation Theorem} to an appropriate work average calculated with an irreversible path. In the Jarzynski scenario, and also in Crooks's generalized FT, the system is initially in thermal equilibrium but then driven out of equilibrium by the action of an external agent. Let $x_{\mathrm{F}}(s)$ denote a time-dependent nonequilibrium "forward" process for which the variable $s$ runs from 0 to some final time $t$. The forward process initially acts on an equilibrium state $A$ and it and ends at a state B that is not at equilibrium. In the reverse process, the initial state B is allowed to reach equilibrium and the system evolves to a nonequilibrium state A. The nonequilibrium protocol for the reverse process $x_{R}(s)$ is time-reversed with respect to the forward one, $x_{R}(s)=x_{F}(t-s)$, so that both processes last for the same time $t$. Let $P_{\mathrm{F}}(W)$ and $P_{\mathrm{R}}(W)$ stand for the work probability distributions along the forward and reversed processes respectively. Then the Crooks FT asserts

$$
\frac{P_{\mathrm{F}}(W)}{P_{\mathrm{R}}(-W)}=\exp \left(\frac{W-\Delta G}{k_{\mathrm{B}} T}\right) .
$$

The Crooks FT can be manipulated to yield the JE. It also resembles the Gallavotti-Cohen FT (equation 3) derived for steady-state systems if one identifies $\sigma t$ with $W_{\text {dis }} / T=(W-\Delta G) / T$. The main difference is that the Gallavotti-Cohen relation is asymptotically valid, whereas the Crooks theorem holds for any finite time $t$. 


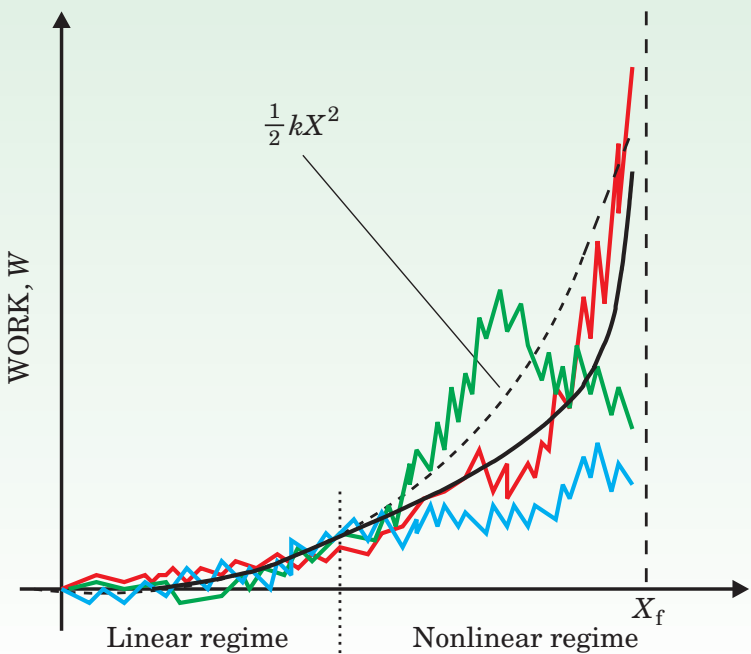

CONTROL PARAMETER, $X$

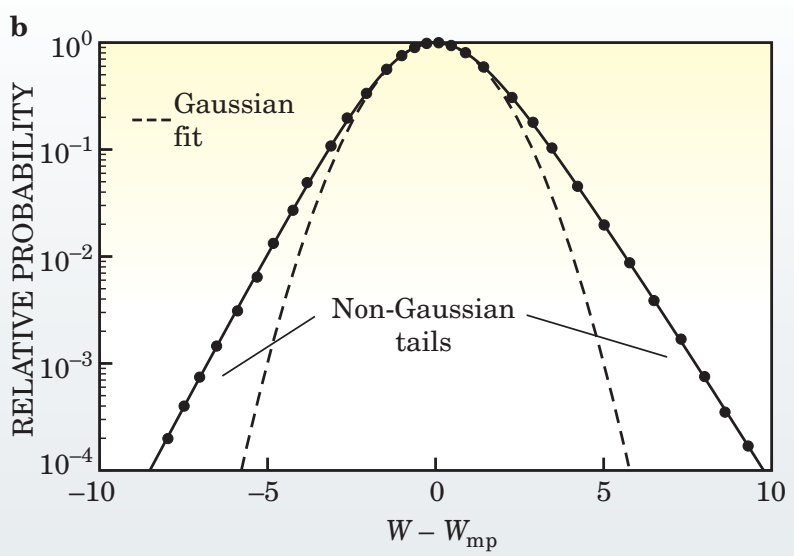

Computer simulations have played an essential role in the development of the FTs. ${ }^{6}$ Indeed, the first paper on the subject, the 1993 report by Evans, Cohen, and Gary Morriss, ${ }^{3}$ included molecular-dynamics simulations of a two-dimensional gas of disks. Over suitably short times, their computer runs showed spontaneous ordering of the gas, in agreement with the expression the authors had derived for the probability of fluctuations of a nonequilibrium steady-state fluid's sheer stress. Computer simulations of nonequilibrium systems continue to be important primarily due to the difficulty of setting up and characterizing suitable nonequilibrium small systems. Conversely, FTs, and especially the JE, can potentially be used to improve the performance of molecular-dynamics simulations.

\section{Experimental tests of fluctuation theorems}

Theorists who consider small systems have greatly benefited from advances in micromanipulation that make it possible to measure energy fluctuations in nonequilibrium small systems. With such measurements, experimenters can test the validity of FTs and scrutinize some fundamental assumptions of statistical mechanics. Sergio Ciliberto and Claude Laroche, in their 1998 study of Rayleigh-Benard convection, performed the first experimental test of the Gallavotti-Cohen FT. ${ }^{7}$ In 2002, Evans's group verified an integrated form of equation 3 in an experiment that used an optical trap to repeatedly drag microscopic beads through water. ${ }^{8}$ They computed the entropy production for each bead trajectory and found that the likelihood of entropy-consuming trajectories relative to entropy-producing trajectories was precisely as theory pre-
Figure 5. The nonlinear regime. (a) The work required to stretch a short polymer is a fluctuating function. The plot shows three different nonequilibrium trajectories obtained as the control parameter $X$ varies from 0 to $X_{f}$. The continuous black line is the work averaged over all trajectories. The dashed line is the linear behavior described by the quadratic function $W=(1 / 2) k X^{2}$. The size of work fluctuations and the deviations of the average work from the dashed line are greater in the nonlinear regime than in the linear regime. (b) In the nonlinear regime, the work probability distribution $P(W)$ has a Gaussian component plus long non-Gaussian tails describing rare processes. Such a mix of Gaussian and non-Gaussian behavior has been seen in the power fluctuations in resistors, the relaxation of glassy systems, Rayleigh-Bernard convection, turbulent flows, and energy fluctuations in granular media. The existence of linear and nonlinear processes occurring along different time scales is reminiscent of the phenomenon of intermittency in turbulent flows and suggests an interesting link between apparently unrelated physical phenomena. In the plot, the zero of the horizontal axis is the most probable $(\mathrm{mp})$ work and the energy unit is $k_{\mathrm{B}} T$. The black dots represent an analytical solution for a nonlinear two-state system, and the solid curve is a non-Gaussian fit. (Adapted from ref. 18.)

dicted. For short times in the millisecond range, the researchers readily observed entropy-consuming trajectories. And as expected, the classical bulk behavior was recovered for longer times on the order of seconds.

Gerhard Hummer and Attila Szabo noted the biophysical relevance of the JE and showed how free energies could be extracted via single-molecule experiments carried out under nonequilibrium conditions. ${ }^{9}$ Soon thereafter, a group led by one of us (Bustamante) tested the JE by mechanically stretching a single molecule of RNA, both reversibly and irreversibly, between its folded and unfolded conformations. ${ }^{10}$ Figure 4 illustrates the group's experimental design. When the RNA was unfolded slowly, the average forward and reverse trajectories could be superimposed; that is, the reaction was reversible. When the RNA was unfolded more rapidly, the mean unfolding force increased and the mean refolding force decreased. The folding-unfolding cycle was thus hysteretic, an indication that work was dissipated. When Bustamante and coworkers applied the JE to the irreversible work trajectories, they recovered the free energy of the unfolding process to within $k_{\mathrm{B}} T / 2$ of its best independent estimate-the work needed to reversibly stretch the RNA. Their experimental test is an example of how the JE bridges the statistical mechanics of equilibrium and nonequilibrium systems.

Experimenters have continued to progress in their ability to test FTs. Technical improvements have recently enabled Evans's group to test equation 3, rather than its integrated form. ${ }^{11}$ Nicolas Garnier and Ciliberto have used electrical circuits as the driven, dissipative system. ${ }^{12}$ They injected current to maintain an electrical dipole, composed of a resistor and a capacitor, in a nonequilibrium steady state; collected the probability distributions of work and heat; and showed those distributions to be in very good agreement with the appropriate FT.

Compared to tests involving trapped beads or stretched polymers, experiments with electrical circuits are less prone to drift and other systematic biases, and they permit much greater numbers of trajectories. Those advantages allow for the investigation of systems with larger dissipation rates. By recording several hours of fluctuation data from their driven electrical dipole, Garnier and Ciliberto investigated the exchanged heat and work with unusually high resolution, and detected the non-Gaussian tails in the heat 


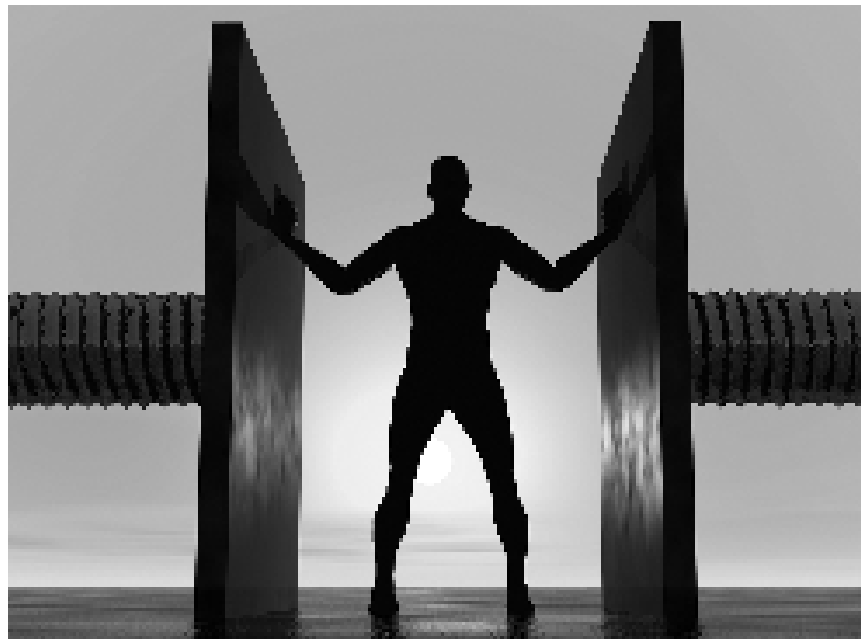

Cost containment pressure squesing efficienoy out of your R\&D?

wiks has youl solutions.

Rising R\&D costs can Imit the bilth of new icleas and the output of tomoliow's best techndojy. To alleviate pressule in the lab. Miks Instiuments deliwers lowcost solutions for monitoing and measuling the vacuum envilonment. Our $\mathrm{HPS}^{\circ}$ ine of vacuum transolucers featules a compact, sensol-on-a-chip clesign that saves space and money. And with a wicle measulement langle, one transolucer can clo the job of seielad.

MKS eVision ${ }^{\text {n1 }}$ RGAs afforclably troubleshoot vacuum, chedr system status. cletect leavs, and monitor chamber gjas acioss a wicle range of apoplications. Togjether; these loks vacuum solutions provicle the highest value at the lowest cost. and let you concentrate mole on biealcthoughes and less on your buclget.
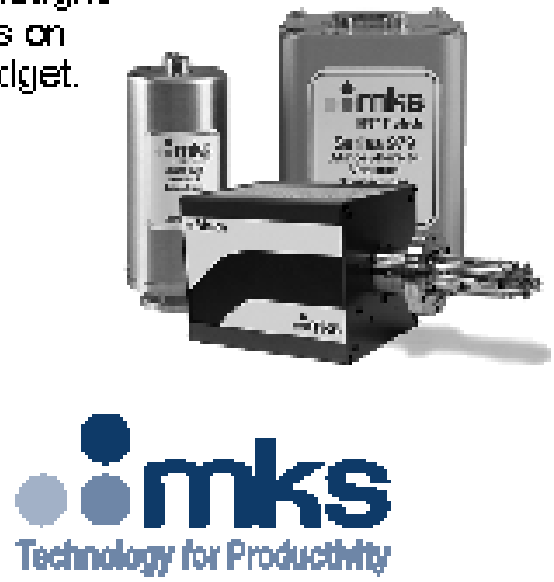

$978.284 .4000 / 800.227 .8760$ (USA only) ' $N$ 'N'N.mirest.com distribution. Such tails, predicted by Ramses van Zon and Cohen for the heat distribution in linear systems, ${ }^{13}$ are also characteristic of work distributions in systems with a nonlinear response to change in a control parameter. For further details see figure 5.

Physicists have also deepened their understanding of the JE. Jarzynski's result-and Crooks's generalization as well-applies to systems that start at equilibrium and are then driven out of equilibrium by some external influence. For many systems of interest, however, including biological molecular machines and nanophotonics devices, the JE does not apply. Such systems often execute irreversible transitions between nonequilibrium steady states. In 1998, Yoshitsugu Oono and Marco Paniconi proposed a general phenomenological framework that encompassed nonequilibrium steady states and transitions between such states. Three years later, Takahiro Hatano and Shinichi Sasa built on that work and generalized the JE to arbitrary transitions between nonequilibrium steady states. ${ }^{14}$ Their result was tested and confirmed last year by a measurement of the dissipation and fluctuations of microspheres optically driven through water. ${ }^{15}$ Those theoretical and experimental advances represent steps toward a complete theory of steady-state thermodynamics. Such a theory would have a profound effect on how scientists describe nonequilibrium steady-state systems such as molecular machines and cells.

Although we have not discussed them, several quantum versions of the classical FTs have appeared in the physics literature. To date, no quantum FT has yet received experimental scrutiny, but such experiments might show interesting surprises. For example, quantum coherence may allow large fluctuations to be observed and FTs to be tested in much larger systems than would be possible in a classical world. That and many other exciting challenges remain for scientists continuing to work with small systems, a fertile ground where physics, chemistry, and biology converge.

\section{References}

1. For a review, see F. Ritort, Séminaire Poincaré 2, 193 (2003), available at http://arXiv.org/abs/cond-mat/0401311.

2. D. J. Evans, D. J. Searles, Phys. Rev. E 50, 1645 (1994); G. Gallavotti, E. G. D. Cohen, Phys. Rev. Lett. 74, 2694 (1995). For a review of fluctuation theorems, see D. J. Evans, D. J. Searles, Adv. Phys. 51, 1529 (2002).

3. D. J. Evans, E. G. D. Cohen, G. P. Morriss, Phys. Rev. Lett. 71, 2401 (1993).

4. C. Jarzynski, Phys. Rev. Lett. 78, 2690 (1997).

5. G. E. Crooks, Phys. Rev. E 60, 2721 (1999).

6. S. Park, K. Schulten, J. Chem. Phys. 120, 5946 (2004).

7. S. Ciliberto, C. Laroche, J. Physique IV 8, 215 (1998).

8. G. M. Wang, E. M. Sevick, E. Mittag, D. J. Searles, D. J. Evans, Phys. Rev. Lett. 89, 050601 (2002).

9. G. Hummer, A. Szabo, Proc. Natl. Acad. Sci. USA 98, 3658 (2001).

10. J. Liphardt, S. Dumont, S. B. Smith, I. Tinoco Jr, C. Bustamante, Science 296, 1832 (2002).

11. D. M. Carberry, J. C. Reid, G. M. Wang, E. M. Sevick, D. J. Searles, D. J. Evans, Phys. Rev. Lett. 92, 140601 (2004).

12. N. Garnier, S. Ciliberto, Phys. Rev. E 71, 060101 (2005).

13. R. van Zon, E. G. D. Cohen, Phys. Rev. E 67, 046102 (2003).

14. Y. Oono, M. Paniconi, Prog. Theor. Phys. Suppl. 130, 29 (1998); T. Hatano, S. Sasa, Phys. Rev. Lett. 86, 3463 (2001).

15. E. H. Trepagnier, C. Jarzynski, F. Ritort, G. E. Crooks, C. Bustamante, J. Liphardt, Proc. Natl. Acad. Sci. USA 101, 15038 (2004).

16. A. M. Fennimore, T. D. Yuzvinsky, W.-Q. Han, M. S. Fuhrer, J. Cumings, A. Zettl, Nature 424, 408 (2003).

17. D. Keller, D. Swigon, C. Bustamante, Biophys. J. 84, 733 (2003).

18. F. Ritort, J. Stat. Mech. P10016 (2004). 